\title{
SIGNO DE LESER-TRÉLAT, SIGNO PARANEOPLÁSICO EN CÁNCER DE MAMA METASTÁSICO: PRESENTACIÓN DE CASO
}

\author{
YURIET ALEXANDRA TIBADUIZA MOGOLLÓN ${ }^{1}$ \\ AUDREY JOSÉ MIRANDA-DIAZ2 \\ DIANA STEFANIA NAVAS TORREJANO \\ ${ }^{1}$ Médico Cirujano Universidad Pedagógica y Tecnológica de Colombia. Tunja. Colombia \\ ${ }^{2}$ Medico. Residente IV año Posgrado Cirugía Plástica. Universidad Industrial de Santander. Bucaramanga. Colombia \\ ${ }^{3}$ Medico. Residente III año Posgrado Medicina Interna. Universidad de Cartagena. Bolívar, Colombia \\ Correspondencia: Yuriet Alexandra Tibaduiza Mogollón \\ alexandratibaduiza@hotmail.com
}

Recibido: 2 de abril de 2018 Aceptado: 24 de julio de 2018

\begin{abstract}
Resumen
El signo de Leser-Trélat es una erupción súbita y de veloz crecimiento de cuantiosas queratosis seborreicas secundarias a una neoplasia interna. Estas pueden estar relacionadas con prurito y acantosis nigricans. Las neoplasias asociadas frecuentemente al signo de Leser-Trélat son los adenocarcinomas gástricos, de colon y mama, representado en menor cantidad por linfomas, carcinoma de células escamosas y malignidades hematológicas. Siendo el signo de Leser-Trélat una dermatosis paraneoplásica infrecuente, se presenta un caso de una paciente de 76 años con cáncer de mama avanzado asociado a este signo.
\end{abstract}

Palabras clave: erupción; queratosis seborreica; prurito; acantosis nigricans; adenocarcinoma, neoplasias de la mama

\section{LESER-TRÉLAT SIGN: PARANEOPLASTIC SIGN IN METASTATIC BREAST CANCER, CASE PRESENTATION}

\begin{abstract}
The Leser-Trelat sign is a sudden and rapid growth eruption of large seborrheic keratoses secondary to an internal neoplasm. These may be related to pruritus and acanthosis nigricans. The neoplasms frequently associated with the Leser-Trélat sign are gastric, colon and breast adenocarcinomas, represented to a lesser extent by lymphomas, squamous cell carcinoma and hematological malignancies. As the Leser-Trélat sign is an uncommon paraneoplastic dermatosis, a case of a 76-year-old patient with advanced breast cancer associated with this sign is presented.
\end{abstract}

Keywords: eruption; seborrheic keratosis; pruritus; acanthosis nigricans; adenocarcinoma, breast neoplasms. 


\title{
SINAL DE LESER-TRÉLAT: SINAL PARANEOPLÁSICO EM CÂNCER DE MAMA METASTÁTICO, APRESENTAÇÃO DE CASO
}

\begin{abstract}
Resumo
O sinal de Leser-Trélat é uma erupção súbita e de veloz crescimento de múltiplas ceratoses seborreicas secundárias a uma neoplasia interna. Estas podem estar relacionadas com prurido e acantose nigricans. As neoplasias associadas frequentemente ao sinal de Leser-Trélat são os adenocarcinomas gástricos, de colón e mama, representado em menor quantidade por linfomas, carcinoma de células escamosas e malignidades hematológicas. Sendo o sinal de LeserTrélat uma dermatose paraneoplásica infrequente, apresenta-se um caso de uma paciente de 76 anos com câncer de mama avançado associado a esse sinal.
\end{abstract}

Palavras-chave: erupção; ceratose seborreica; prurido; acantose nigricans; adenocarcinoma, neoplasias da mama.

\section{Introducción}

Los síndromes paraneoplásicos cutáneos pertenecen al grupo de manifestaciones en piel que se evidencian antes, durante o posterior al debut de una enfermedad tumoral con la que mantienen una relación de dependencia no metastásica (1).

El signo de Leser-Trélat (SLT) se caracteriza por la erupción súbita y de rápido crecimiento de numerosas queratosis seborreicas (QS) secundarias a una neoplasia interna (2-5). Estas lesiones pueden estar asociadas a prurito y más frecuentemente a acantosis nigricans (4). Las neoplasias asociadas con mayor frecuencia al SLT son el adenocarcinoma gástrico, de colon y mama, y en menor medida linfoma/leucemia, carcinoma de células escamosas y malignidades hematológicas $(1,3)$.

Considerándose el SLT como una dermatosis paraneoplásica infrecuente se presenta un caso de una paciente de 76 años con SLT asociado a cáncer de mama avanzado.

\section{Presentación de caso}

Paciente femenina de 76 años, hipertensa crónica en tratamiento con losartán $50 \mathrm{mg}$ cada $12 \mathrm{~h}$, Braden 13, hace 3 meses es sometida a mastectomía radical derecha y vaciamiento regional por carcinoma lobulillar in situ, grado histológico III, T4 N1Aaxila M1. Consulta por presentar cuadro de aproximadamente 1 semana de evolución de dolor sin localización específica en miembros inferiores, asociado a sensación desagradable e incontrolable de mover las piernas a pesar de su clasificación de Braden.
Al examen físico se encuentra paciente en malas condiciones generales, consciente orientada, álgica y con notable intranquilidad. Los signos vitales tensión arterial de 100/60, frecuencia cardiaca de 90 latidos por minuto, frecuencia respiratoria 20 por minuto, temperatura de $36,7^{\circ} \mathrm{C}$, IMC 16. Pupilas isocóricas normorreactivas a la luz, no ingurgitación yugular, campos pulmonares sin agregados audibles, ruidos cardiacos con soplo grado I auscultable en región axilar. Como hallazgos anormales, se encuentra en boca lengua depapilada y además múltiples lesiones queratósicas, seborreicas e hiperpigmentadas en gran parte de su superficie corporal que incluye cuello, tórax, abdomen y extremidades inferiores (figuras 1 y 2).

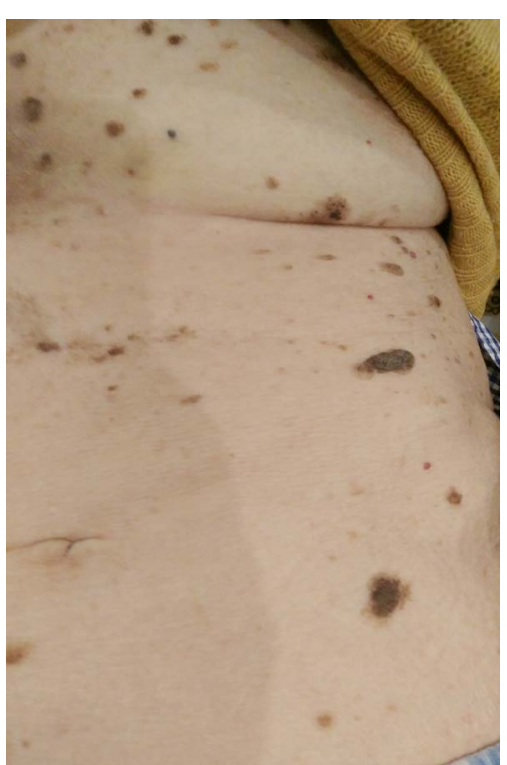

Fotografia 1. Míltiples queratosis seborreica en abdomen y torax. Fuente: Fotografía de los autores. 


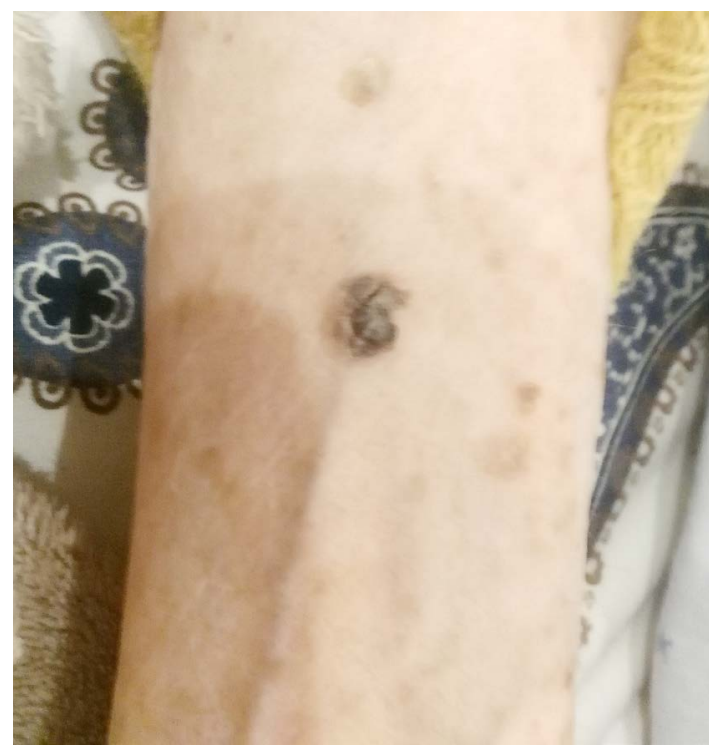

Fotografia 2. Queratosis seborreica de $1 \times 1 \mathrm{~cm}$ en cara anterior tercioproximal de antebrazo.

Fuente: Fotografía de los autores.

Cicatriz de mastectomía en buenas condiciones y en adecuado proceso de cicatrización.

Se decide solicitar exámenes de laboratorio en la paciente y se encontraron concentraciones séricas de sodio, potasio y cloro normales. Parcial de orina sin alteraciones. Hemograma: hematocrito del $40 \%$, hemoglobina del 10\%, leucocitos de 12 100, vcm de $70 \mathrm{fl}$, hcm 28 $\mathrm{pg} / c e ́ l u l a$. Se decide solicitar exámenes adicionales y se encontró ferritina de $45 \mathrm{mg} / \mathrm{l}$ e indice de saturación de transferrina del 25\%, hierro de $44 \mathrm{ug} / 100 \mathrm{ml}$.

Se considera paciente con SLT asociado a cáncer de mama avanzado, anemia de la enfermedad crónica, secundaria a su cáncer metastásico, clínicamente evidenciada como sindrome de las piernas inquietas, para lo cual se indica tratamiento con gabapentina y $65 \mathrm{mg}$ de hierro elemental/día, asociado a vitamina C (lejos de los alimentos).

\section{Discusión}

El cáncer de mama es la neoplasia maligna más común en mujeres y corresponde a la segunda causa de muerte por cáncer en el mundo, después del cáncer de pulmón (5). Es diagnosticado en un $81 \%$ entre mujeres de 50 años o más, con una mortalidad del $89 \%$ (6).
El cáncer de mama puede presentar síntomas cutáneos, ya sea por extensión directa, metástasis o secundariamente, como un síndrome paraneoplásico. El 5-10\% de las pacientes en el momento del diagnóstico ya tienen metástasis, entre ellas es la neoplasia maligna que más frecuentemente presenta metástasis a piel, con una incidencia del $20 \%$ según serie de autopsias, debutando cutáneamente en el 3\% de los casos de cáncer de mama metastásico (7).

Las dermatosis paraneoplásicas simbolizan un conjunto diverso de manifestaciones clínicas que surgen como expresión de las acciones a distancia que puede formar una neoplasia subyacente (8). Estas tienen un importante segundo lugar de frecuencia entre las expresiones paraneoplásicas de modo que son despuntadas solo por las de origen endocrinológico (9).

Es conocido que entre un 7 y $15 \%$ de los tumores desarrollaran síndromes paraneoplásicos $(10,15)$ y que un $1 \%$ de los cánceres ostentan como primera manifestación una lesión cutánea (11-12).

La definición clásica procede de los criterios que Curth postuló en 1976 y que hoy por hoy, con algunas modificaciones, continúan vigentes para establecer la asociación entre la dermatosis y la neoplasia: a) comienzo simultáneo: ambas enfermedades se desarrollan aproximadamente a la vez; b) evolución paralela: desaparición de la enfermedad cutánea cuando se trata el tumor y reaparición con recidiva o metástasis; c) asociación estadísticamente significativa; d) rareza de la dermatosis (la enfermedad cutánea per se es poco frecuente); y e) malignidad uniforme: un mismo tipo de tumor debe provocar una misma manifestación cutánea $(8,9)$.

Los síndromes paraneoplásicos cutáneos reflejan la interacción existente entre las células tumorales tanto del tumor primitivo como de sus metástasis y el huésped. La patogenia de estos procesos no se ha dilucidado totalmente, pero se atribuye a la producción excesiva o a la depleción de sustancias biológicamente activas, factores de crecimiento, hormonas, otros mediadores no identificados producidos por el tumor o a interacciones antígeno-anticuerpo con una respuesta aberrante por parte del huésped a varios tipos de cáncer $(10,13)$.

La queratosis seborreica pertenece a lesiones cutáneas, con patrón aislado o diseminado, que comprometen usualmente la cara, el tronco y los miembros superiores. 
Se muestra primariamente como pápulas pequeñas de $1 \mathrm{a}$ $3 \mathrm{~mm}$ de diámetro que evolucionan a placas bien circunscritas e hiperpigmentadas de 1 a $6 \mathrm{~cm}$ de diámetro, con superficie verrugosa de aspecto oleoso y con quistes córneos en la dermatoscopia. Generalmente aparecen luego de los 50 años y siguen presentes durante toda la vida, y acrecienta su dispersión a medida que se envejece (15). El concepto del SLT implica la aparición brusca y el veloz desarrollo en el tamaño y número de las QS (16); a pesar de los estudios, aún no hay criterios universales en cuanto al número de lesiones y tiempo de evolución. En este sentido, Lindelöff et al. (15) definen el intervalo de tiempo en 3 a 18 meses para la aparición de 20 o más lesiones. Se estima que el tiempo medio de instauración de las lesiones dermatológicas es 15 semanas (3). La localización de las QS puede comprometer cualquier localización cutánea, con un patrón típicamente denominado en árbol de $\mathrm{Na}$ vidad, de modo que es más frecuente en el tronco (40\%), seguido de las extremidades $(10,8 \%)(3,13)$. El prurito puede ser una característica prominente en el $26-51 \%$ de los casos (15). Incluso en pacientes con cáncer su frecuencia es rara, no tiene predisposición racial ni por género.

Se ignora el mecanismo patogénico exacto de este síndrome (16), existen diversas hipótesis, pero se considera la producción de múltiples citocina y factores de crecimiento como factor de crecimiento epidérmico el cual se encuentra sobreactivado y el factor transformador del crecimiento alfa, estructuralmente relacionado, pero antigénicamente distinto del factor de crecimiento epidérmico $(2-4,17)$. Estos factores de crecimiento se unen a sus receptores cutáneos, estimulan la proliferación de los queratinocitos y alteran el tejido de la matriz extracelular, lo que posiblemente deriva en la formación de queratosis seborreica (18). Ellis et al. (15) demostraron en un paciente con síndrome de Leser-Trélat asociado a melanoma cutáneo un aumento difuso de los receptores del factor de crecimiento epidérmico en todas las capas del estrato mucoso de Malpighi, mientras que en condiciones normales este receptor se localiza exclusivamente en los queratinocitos basales, así como un declive en el número de receptores al llevar a cabo la extirpación del tumor. En ciertos pacientes, se han identificado altos niveles de hormona de crecimiento humana inmunorreactiva. Estas hipótesis explicarían la consecuente frecuencia de hasta un $35 \%$ de los casos con la que coexisten el SLT y la acantosis nigricans, ya que, en ambos casos, la base histopatológica es igualmente una hiperplasia epidérmica $(1-3,5)$, de modo que es una de varias de las características que apoyan su legitimidad como un verdadero trastorno paraneoplásico (15), así como su descripción en pacientes jóvenes con cáncer subyacente, pese al debate generado debido a la aparición frecuente de queratosis seborreica en los ancianos (17).

El pronóstico de los pacientes que presentan SLT depende habitualmente del tipo de neoplasia asociada (15), y para su tratamiento siempre la mejor alternativa es el control de la neoplasia asociada, aunque se ha documentado el uso de los retinoides tópicos, ácido tricloroacético, dermoabrasión, láser o criocirugía, con curetaje o sin él para el control de las queratosis (6).

\section{Conclusiones}

La presencia de lesiones seborreicas con aparición repentina, lesiones pigmentadas y en gran número deben alertar al clínico para descartar otras entidades y asociaciones a otras lesiones como neoplasias de forma temprana ( 9 , 17, 19). En casos de duda diagnóstica, la biopsia de las lesiones dérmicas confirma el diagnóstico de QS (3).

\section{Conflictos de interés}

Los autores declaran no tener de manera directa o indirecta ningún tipo de conflicto de intereses financieros, académicos o laborales que puedan poner en riesgo la validez de este estudio.

\section{Referencias}

1. Venegas F, Vaccaro P, Abudinén G, et al. Signo de LeserTrélat asociado a adenocarcinoma gástrico: caso clínico. Rev Med Chile. 2012;140(12):1585-1588.

2. Blázquez N, Fernández-López E, Fernández-Canedo I, et al. Signo de Leser-Trélat asociado a síndrome de Sézary. Actas Dermosifiliogr. 2003;94(5):309-312.

3. Martínez-Hernández P, Báez-García J, Báez-Aviña J. Signo de Leser-Trélat con carcinoma de recto. Rev Gastroenterol Mex. 2014;79(4):294-295.

4. Mohanna S, Bravo F, Salinas E, Solidoro A. Signo de LeserTrélat en una paciente con adenocarcinoma de pulmón. Folia dermatol. Peru, 2005;16(3):123-126.

5. Gradishar WJ, Anderson BO, Balassanian R, et al. NCCN guidelines insights: breast cancer, version 1.2017. J Natl Compr Canc Netw. 2017;15(4):433-451.

6. DeSantis CE, Ma J, Sauer AG, et al. Breast cancer statistics, 2017, racial disparity in mortality by state. CA Cancer J Clin. 2017;67(6):439-448.

7. Tan AR. Cutaneous manifestations of breast cancer. Semin Oncol. 2016;43(3): 331-334.

8. Rivera MAA, López HMA. Dermatosis paraneoplásicas: revisión de la bibliografía. Med Int Mex. 2011;27(6):586595. 
9. Venegas F, Vaccaro P, Abudinén G, et al. Signo de LeserTrélat asociado a adenocarcinoma gástrico: Caso clínico. Rev Med Chile. 2012;140(12):1585-1588.

10. Yuste-Chaves M, Unamuno-Pérez P. Alertas cutáneas en malignidades sistémicas (parte I). Actas Dermosifiliogr. 2013;104(4):285-298.

11. Ahuja S, Makkar P, Gupta S, et al. Paraneoplastic syndrome and underlying breast cancer: a worsening rash despite initiation of chemotherapy. J Community Support Oncol. 2016;14(5):229-231.

12. Millán GG, Sánchez CA, García LE, et al. Síndromes paraneoplásicos cutáneos. Semergen. 2004;30(10):506-513.

13. Martínez-Morán C, Sanz-Muñoz C, Miranda-Romero A. Signo de Leser-Trélat asociado a sindrome de Sézary ya carcinoma de células transicionales de vejiga. Actas Dermosifiliogr. 2007;98(3):214-215.
14. Chung VQ, Moschella SL, Zembowicz A, et al. Clinical and pathologic findings of paraneoplastic dermatoses. J Am Acad Dermatol. 2006;54(5), 745-762.

15. Mantilla AF, Díaz-Martínez LA, Ballesteros ZJ, et al. El signo de Leser-Trélat: ¿un predictor de neoplasias útil en clínica? Acta Med Colomb. 2014;39(3):272-278.

16. Al Ghazal P, Körber A, Klode J, et al. Leser-Trélat sign and breast cancer. The Lancet, 2013;381(9878):1653.

17. Chakradeo K, Narsinghpura K, Ekladious A. Sign of LeserTrélat. BMJ Case Rep. 2016;8:2016.

18. Schwartz R. Sign of Leser-Trélat. Medscape [internet]. 2018 abr [citado 0219 mrz 14]. Disponible en: https:// emedicine.medscape.com/article/1097299-overview\#a6

19. Sardon C, Dempsey T. The Leser-Trélat sign. Cleve Clin J Med. 2017;84(12):918-918. 